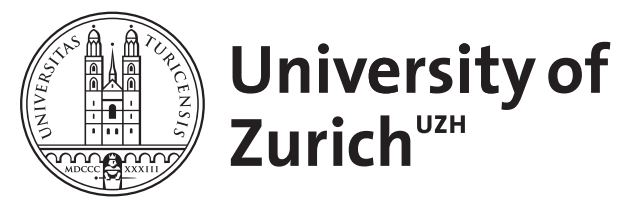

\title{
Die Therapie des komplizierten Morbus Crohn
}

\author{
Scharl, Michael ; Barthel, Christiane ; Rogler, Gerhard
}

\begin{abstract}
During their disease course, the majority of Crohn's disease patients will develop a complicated disease which is characterized by the occurrence of fistulas and/or stenosis. Symptomatic, perianal fistulas should be surgically drained before anti-inflammatory therapy will be initiated. Antibiotics, such as metronidazole, improve disease symptomatic however, they are not sufficient to induce continuous fistula closure. For this purpose, azathioprine/6-mercaptopurine as well as anti-TNF antibodies are useful when administered continuously. Surgical options include seton drainage, fistula excision, fistula plugs and mucosa flaps. As ultima ratio, temporary ileostomy and proctectomy are to be discussed. Non-perianal fistulas often require surgical approaches. Symptomatic strictures or stenosis can be treated by antiinflammatory medications (only if they are cause by inflammation), endoscopic balloon dilatation or surgery.
\end{abstract}

DOI: https://doi.org/10.1024/1661-8157/a001592

Other titles: Therapy of complicated Crohn's disease

Posted at the Zurich Open Repository and Archive, University of Zurich

ZORA URL: https://doi.org/10.5167/uzh-105064

Journal Article

Accepted Version

Originally published at:

Scharl, Michael; Barthel, Christiane; Rogler, Gerhard (2014). Die Therapie des komplizierten Morbus Crohn. Praxis, 103(6):323-327.

DOI: https://doi.org/10.1024/1661-8157/a001592 
${ }^{1}$ Klinik für Gastroenterologie und Hepatologie, Universitätsspital Zürich

${ }^{2}$ Klinik und Poliklinik für Innere Medizin, Universitätsspital Zürich

PD Dr. med. Michael Scharl ${ }^{1,2}$, Dr. med. Christiane Barthel ${ }^{1}$, Prof. Dr. med. Dr. phil. Gerhard Rogler ${ }^{1}$

Die Therapie des komplizierten Morbus Crohn

Therapy of complicated Crohn's disease

Abkürzungen

European Crohn's and Colitis Organisation, ECCO; Morbus Crohn; Tumor Nekrose Faktor, TNF. 


\section{Zusammenfassung}

Bei einem signifikanten Teil der Morbus Crohn Patienten entwickelt sich im Krankheitsverlauf Komplikationen, wie das Auftreten von Fisteln und/oder Stenosen. Symptomatische, peri-anale Fisteln sollten zunächst drainiert werden, bevor eine anti-entzündliche Behandlung eingeleitet wird. Antibiotika, wie Metronidazol, wirken zunächst symptomatisch, ein dauerhafter Fistelverschluss wird hiermit jedoch nicht erreicht. Hierfür stehen Azathioprin/6-Mercaptopurin sowie insbesondere anti-TNF Antikörper zur Verfügung. Diese Medikamente führen bei kontinuierlicher Gabe bei rund 50\% der Patienten sowohl zu einem initialen als auch andauernden Fistelverschluss. Chirurgisch kann eine Versorgung mittels Seton-Faden, Fistelexzision, Fistel-Plug oder Mukosa-Flap durchgeführt werden. Als ultima ratio kommen eine temporäre lleostomie oder eine Rektumresektion infrage. Nicht-peri-anale Fisteln erfordern häufig ein chirurgisches Vorgehen. Stenosen können mittels anti-entzündlicher Medikamente (wenn sie entzündlicher Genese sind), Ballondilatation oder Chirurgie behandelt werden.

During their disease course, the majority of Crohn's disease patients will develop a complicated disease which is characterized by the occurrence of fistulas and/or stenosis. Symptomatic, perianal fistulas should be surgically drained before anti-inflammatory therapy will be initiated. Antibiotics, such as metronidazole, improve disease symptomatic however, they are not sufficient to induce continuous fistula closure. For this purpose, azathioprine/6-mercaptopurine as well as anti-TNF antibodies are useful when administered continuously. Surgical options include seton drainage, fistula excision, fistula plugs and mucosa flaps. As ultima ratio, temporary ileostomy and proctectomy are to be discussed. Non-perianal fistulas often require surgical approaches. Symptomatic strictures or stenosis can be treated by anti-inflammatory medications (only if they are cause by inflammation), endoscopic balloon dilatation or surgery.

Chez la majorité des patients atteints par la maladie de Crohn se dévelope un déroulement complexe de la maladie; ce dernier est marque par des fistules et/ou sténoses. Les fistules perianales devraient être drainées avant de commencer un traitement anti-inflammatoire. Les antibiotiques, comme Metronidazol, agissent d'abord symptomatiquement, mais on ne parvient pas à une fermeture permanente de la fistule. Pour ceci, il y a Azathioprin/6-Mercaptopurin et surtout des anticorps anti-TNF. Ces médicaments mènent à une fermeture à la fois initiale et 
permanente de la fistule. Il est également possible de mettre chirurgicalement une ficelle Seton ou encore d'effectuer une iléostomie temporaire ou une resection rectale antrieure. Des fistules non-perianales nécessitent souvent la voie chirurgicale. Des sténoses peuvent être traitées avec des médicaments anti-inflammatoires, dilatation de ballon ou bien chirurgicalement. Idéalement les cas des patients atteint de fistules et de sténoses devraient être discutes et traites interdisciplinairement.

\section{Schlüsselwörter}

Morbus Crohn, Fisteln, chronisch-entzündliche Darmerkrankung

Crohn's disease, fistulae, inflammatory bowel disease

Maladie de Crohn, fistule, maladies inflammatoires chroniques 


\section{Einleitung}

Rund 15'000 Menschen in der Schweiz leiden an einer Form einer chronisch-entzündlichen Darmerkrankungen. Etwa die Hälfte dieser Patienten leiden an Morbus Crohn (MC). Bei Diagnosestellung präsentiert sich die Mehrheit der Patienten mit einem entzündlichen (inflammatorischen) Erkrankungstyp. Im Laufe der Erkrankung tritt jedoch - zumindest bei Patienten an Zentren - zunehmend ein stenosierender oder fistulierender Krankheitstyp auf (1). Insgesamt entwickeln rund $35 \%$ der MC Patienten im Laufe ihres Lebens eine oder mehrere Fisteln. Hiervon entfallen $54 \%$ auf peri-anale Fisteln, $24 \%$ auf entero-enterische Fisteln, 9\% auf rekto-vaginale Fisteln sowie $13 \%$ auf Fisteln anderer Lokalisationen, wie etwa entero-kutan, entero-vesikal oder intra-abdominell (2). Die kumulative Inzidenz für das Auftreten von Fisteln lag in dieser Studie bei $33 \%$ nach 10 Jahren sowie $50 \%$ nach 20 Jahren Krankheitsdauer. Rund ein Drittel der Patienten leiden unter rezidivierenden Fisteln (2) und etwa 20-30\% der MC Patienten weisen peri-anale Läsionen oder Fisteln bei der Erstdiagnose auf (1).

\section{Symptomatik, Einteilung, Entstehung und Diagnostik von Fisteln}

Fisteln können sich mit kontinuierlichen Schmerzen im Analbereich, schmerzhafter Defäkation sowie peri-analen Öffnungen mit eitrigem Ausfluss manifestieren. Die Fistelöffnungen sind häufig im Bereich der Analhaut lokalisiert, finden sich jedoch gelegentlich auch im Bereich des Skrotum, der Vulva oder der Leiste. Aufgrund ihrer anatomischen Ausdehnung können perianale Fisteln in einfache und komplexe Fisteln unterteilt werden. Einfache Fisteln sind in der linea dentata lokalisiert, weisen eine einzige Fistelöffnung auf, sind nicht schmerhaft und zeigen keinen Hinweis auf rekto-vaginale Fisteln oder ano-rektale Strikturen. Im Gegensatz dazu liegen komplexe Fisteln oberhalb der linea dentata, haben zahlreiche Fistelöffnungen, zweigen Hinweise auf einen Abszess und können schmerzhaft oder mit einer rekto-vaginalen Fistel, einer ano-rektalen Striktur oder endoskopisch aktivem, rektalen MC vergesellschaftet sein (3). Das Risiko für das Auftreten von Fisteln ist in MC Patienten mit Kolonbefall und insbesondere bei Patienten mit Befall des Rektums höher als in Patienten ohne kolorektalen Befall (1).

Dies passt zur Hypothese, dass Fisteln aufgrund einer kontinuierlichen Gewebszerstörung im Rahmen der chronischen, transmuralen Entzündung des $M C$ entstehen. Infektionen führen zu einem Abszess in der Darmwand und schliesslich zu einem Eindringen des Abszessgewebes nach seiner spontanen Drainage in angrenzende Organe. Für das Vordringen der Fisteln in das 
Gewebe dürfte dann die sogenannte epitheliale-mesenchymale Transition (EMT) auf molekularer Ebene eine wichtige Rolle spielen. Hierbei verwandeln sich ortsständige, differenzierte Darmschleimhautzellen in entdifferenzierte, wanderungsfähige Mesenchymalzellen. Derart veränderte Zellen können in den Fistelgängen eindeutig nachgewiesen werden $(4,5)$. Die Diagnostik der Fisteln basiert zum einen auf der klinischen Untersuchung (gegebenenfalls unter Anästhesie) und zum anderen auf der Bildgebung mittels kontrastmittel-verstärkter Magnetresonanztomographie (insbesondere bei peri-analen und/oder komplexen Fisteln) und endoskopischer, ano-rektaler Sonographie. Zudem können die Computertomographie, Endoskopie sowie die transkutane Sonographie hilfreich sein. Eine direkte Fistulographie sollte hingegen nicht durchgeführt werden (6).

\section{Therapie}

Die Behandlung von Fisteln beinhaltet sowohl medikamentöse wie auch chirurgische Ansätze. Asymptomatische, einfache Fisteln müssen nicht zwingend behandelt werden (6). Interessanterweise kommt es in rund $10 \%$ der Fälle zu einem spontanen Fistelverschluss, wie anhand einer Metaanalyse placebo-kontrollierter Studien gezeigt werden konnte (7). Für symptomatische, peri-anale, einfache Fisteln stehen die Einlage eines Seton-Fadens sowie eine Fistulotomie mit anschliessender Antibiotikabehandlung, etwa mit Ciprofloxacin oder Metronidazol, zur Verfügung. Bei komplexer, peri-analer Erkrankung sollten Abszesse generell drainiert und mittels Seton-Einlage versorgt werden. Zudem sollte eine medikamentöse Therapie mittels Antibiotika und Immunsuppressiva, erfolgen (6). Als chirurgische Therapieoptionen stehen ein Fistel-Plug sowie ein Mukosa-Flap zur Verfügung. Eine Fistulotomie oder Fistulektomie sollte aufgrund der Gefahr einer Sphinkterverletzung und konsekutiver Inkontinenz nur mit grosser Vorsicht durchgeführt werden. Zudem kann eine Fistel-Kralle, also der Verschluss des Fistelganges durch einen modifizierten Metallklip erwogen werden. Bei schweren, rezidivierenden Fällen kann auch eine Stomaanlage oder eine Rektumresektion nötig sein (6). In der Behandlung komplexer peri-analer Fisteln sollte stets eine Seton-Einlage erfolgen. Zudem stehen verschiedene medikamentöse Ansätze zur Verfügung. Hierzu zählen insbesondere Antibiotika, Azathioprin/6-Mercaptopurin und anti-TNF Antikörper (Abbildung 1). Kortikosteroide und Aminosalicylate sollten hingegen nicht für die Fisteltherapie verwendet werden (8). 


\section{Antibiotika}

Die Behandlung mit Antibiotika, wie Ciprofloxacin und/oder Metronidazol, führt zwar oftmals zu einer Verbesserung der Symptomatik, jedoch kaum zu einem Abheilen der Fisteln. Zudem treten die Beschwerden nach Absetzen des Antibiotikums häufig wieder auf. Am weitesten verbreitet für die Behandlung von Fisteln ist Metronidazol. Nach 6-8 Wochen kommt es häufig zu einer deutlichen Verbesserung der Symptomatik. Allerdings ist die dauerhafte Anwendung durch Nebenwirkungen, wie Übelkeit, Geschmacksveränderungen und das Auftreten peripherer Neuropathien limitiert (9). Über die Wirksamkeit einer Monotherapie mit Ciprofloxacin, wie auch mit Metronidazol, bei peri-analem MC liegen jedoch nur sehr kleine Studien vor. Im Rahmen einer doppelblinden, placebo-kontrollierten Studie konnte jedoch gezeigt werden, dass Ciprofloxacin in Kombination mit Infliximab effektiver in der Behandlung peri-analer Fisteln ist, als die Behandlung mit Infliximab alleine (10).

\section{Azathioprin/6-Mercaptopurin}

Für diese beiden Immunsuppressiva gibt es keine Daten von randomisierten, kontrollierten klinischen Studien. Anhand einer Metaanalyse von 5 Studien konnte jedoch gezeigt werden, dass Azathioprin und 6-Mercaptopurin bei rund $30 \%$ der Patienten sowohl zu einem initialen als auch dauerhaften Fistelverschluss führen (11). Nichtsdestotrotz werden Antibiotika und Azathioprin/6-Mercaptopurin gemäss den aktuellen Guidelines der European Crohn's and Colitis Organisation (ECCO) in Kombination mit einer chirurgischen Behandlung als Therapie der Wahl bei Patienten mit komplexem peri-analem MC empfohlen (6).

\section{Anti-TNF Antikörper}

Die Wirksamkeit von Infliximab bei Patienten mit peri-analen Fisteln konnte im Rahmen randomisierter Studien gezeigt werden. Infliximab ist sowohl bei der initialen wie auch der dauerhaften Fistelheilung wirksam. Jedoch kommt es in rund $50 \%$ der Patienten nach Absetzen des Medikamentes wieder zu einer erneuten Fistelproblematik. Die Behandlung mit Infliximab in einer Dosierung von $5 \mathrm{mg} / \mathrm{kg}$ Körpergewicht verabreicht in den Wochen 0, 2 und 6 induzierte einen kompletten Fistelverschluss bei $55 \%$ der Patienten mit einfachen wie auch komplexen Fisteln (12). In einer Folgestudie zeigte sich bei $69 \%$ der Patienten ein initiales 
Ansprechen nach 14 Behandlungswochen. Diese Patienten erhielten anschliessend entweder Placebo oder $5 \mathrm{mg} / \mathrm{kg}$ Körpergewicht Infliximab alle 8 Wochen. Nach 54 Wochen war bei $36 \%$ der Patienten in der Infliximab-Gruppe immer noch ein kompletter Fistelverschluss nachweisbar. In der Placebogruppe war dies hingegen nur bei $19 \%$ der Fall $(p=0.009)$. Ein Ansprechen, also ein mehr als $50 \%$ Fistelverschluss anhand der klinischen Untersuchung, war ebenfalls signifikant häufiger in der Infliximab-Gruppe nachweisbar (13). Die Behandlung mit Adalimumab führt ebenfalls signifikant häufiger zu einem Fistelverschluss im Vergleich zu Placebo. Die Therapie mit Adalimumab führte bei $30 \%$ der Patienten zu einem Fistelverschluss sowohl nach 26 als auch nach 56 Behandlungswochen. Dies war nur bei $13 \%$ der Patienten in der Placebogruppe der Fall $(p<0.04)$. Bei $90 \%$ der Patienten, die nach 56 Behandlungswochen einen vollständigen Fistelverschluss aufwiesen, hielt dieser unter Adalimumab-Therapie auch noch für ein weiteres Jahr an $(14,15)$. Im Rahmen einer open-label Phase IV Studie konnten Vavricka et al. ebenfalls die Wirksamkeit von Certolizumab pegol bei 60 Patienten mit fistulierendem MC nachweisen. In dieser Studie kam es nach 6 Behandlungswochen bei $36 \%$ und nach 26 Behandlungswochen bei $55 \%$ der Patienten zu einem kompletten Verschluss der peri-analen Fisteln (16). Anti-TNF-Antikörper können in der Behandlung komplexer, peri-analer Fisteln sowohl als erste Wahl als auch zweite Wahl nach Therapieversuch mit Azathioprin/6Mercaptopurin eingesetzt werden (6). Um die Wirksamkeit der anti-TNF Antikörper zu erhöhen, ist eine Kombination mit Ciprofloxacin oder Azathioprin/6-Mercaptopurin möglich.

\section{Methotrexat, Ciclosporin und Tacrolimus}

In einer retrospektiven Studie zeigte sich ein Nutzen für die Behandlung des peri-analen MC mit Methotrexat. Bei 16 Patienten, bei denen 6-Mercaptopurin aufgrund von Wirkungslosigkeit oder Unverträglichkeit pausiert werden musste, konnte durch die Gabe von Methotrexat in 4 Fällen ein kompletter Fistelverschluss und in 5 Fällen ein teilweiser Fistelverschluss erreicht werden (17). Unkontrollierte Fallserien zeigen, dass Ciclosporin und Tacrolimus ebenfalls erfolgreich für die Behandlung des peri-analen, fistulierenden MC eingesetzt werden können. Die CiclosporinGabe führt zu einem guten Kurzzeit-Effekt, welcher jedoch nach Beendigung der Therapie sehr schnell endet (18). Ähnliches gilt für die Behandlung mit oralem Tacrolimus. Hier kommt es ebenfalls initial zu einem Ansprechen, jedoch erlischt der Therapieerfolg rasch nach Absetzen des Medikamentes (19). Aufgrund der signifikanten Nebenwirkungen bei Langzeittherapie ist der Einsatz von Methotrexat, Ciclosporin und Tacrolimus jedoch in der Behandlung des peri- 
analen MC limitiert (6). Ähnliches gilt für enterale oder parenterale Ernährung, Mycophenolat mofetil, Thalidomid, GM-CSF oder die hyperbare Sauerstofftherapie, welche von der ECCO nicht für den klinischen Alltag empfohlen werden (6).

\section{Erhaltungstherapie und Behandlung nicht-peri-analer Fisteln}

Nach dem Fistelverschluss wird eine Erhaltungstherapie über mindestens ein Jahr empfohlen. Hierzu eignen sich entweder Azathioprin/6-Mercaptopurin, Infliximab, Adalimumab, eine SetonDrainage oder eine Kombination aus medikamentöser Behandlung und Drainage (6). Für die Behandlung nicht-peri-analer Fisteln bei MC Patienten fehlen randomisierte Studien. Die adäquate Behandlung dieser Art von Fisteln stellt eine interdisziplinäre Herausforderung für Gastroenterologen, Chirurgen und Radiologen dar. Oft ist eine Resektion des betroffenen Darmsegmentes unerlässlich. Wenn zugleich eine aktive, insbesondere im Bereich des Rektums lokalisierte Entzündung besteht, sollte diese vor der Resektion medikamentös an behandelt werden (6).

\section{Therapie des fibrostenosierenden Morbus Crohn}

Im Rahmen des fibro-stenosierenden $M C$ kommt es zu relevanten Störungen der Darmpassage. Zur Behandlung dieser Stenosen stehen anti-entzündliche Medikamente, die endoskopische Ballondilatation sowie die Chirurgie zur Verfügung. Bei Auftreten der Symptomatik sollte zunächst anhand klinischer, labor-chemischer (z. B. CRP, Calprotectin) und radiologischer Befunde beurteilt werden, ob eventuell eine entzündliche, eine gemischt entzündlich-fibrotische oder eine rein fibrotische Stenose vorliegt. Bei Verdacht auf eine entzündlich bedingte Stenose sollte ein kurzzeitiger Therapieversuch mit Steroiden durchgeführt werden. Falls hiermit kein Erfolg eintritt, kann ein weiterer Versuch mit Infliximab gemacht werden. Ein Therapieversagen spricht für das Vorliegen einer fibrotischen Striktur und gegen eine durch die akute Entzündung verursachte Stenose. In diesem Fall steht zum Einen die endoskopische Ballondilatation und zum Anderen die chirurgische Behandlung mittels Strikturoplastik oder Resektion des betroffenen Darmabschnittes zur Verfügung (20). Falls aufgrund der initialen Diagnostik der hochgradige Verdacht auf eine fibrotische Stenose besteht, ist eine medikamentöse Therapie nicht sinnvoll und es sollte direkt eine Ballondilatation oder chirurgische Therapie durchgeführt werden (Abbildung 2) (20). 


\section{Zusammenfassung}

Die Mehrheit der MC Patienten leidet im Laufe ihrer Erkrankung an Fisteln oder Stenosen. Symptomatische, peri-anale Fisteln und Abszesse sollten zunächst drainiert werden, bevor eine anti-entzündliche Behandlung eingeleitet wird. Antibiotika wirken zunächst symptomatisch, ein dauerhafter Fistelverschluss wird hiermit jedoch nicht erreicht. Hierfür stehen Azathioprin/6Mercaptopurin sowie insbesondere anti-TNF Antikörper zur Verfügung. Stenosen können mittels anti-entzündlicher Medikamente, Ballondilatation oder Chirurgie behandelt werden. Im Idealfall sollten Patienten mit Fisteln und Stenosen, insbesondere an speziellen Zentren, interdisziplinär besprochen und behandelt werden. 


\section{Key messages}

Bei der Mehrheit der Morbus Crohn Patienten entwickelt sich im Laufe ihrer Erkrankung ein komplizierter Krankheitsverlauf, welcher durch das Auftreten von Fisteln und/oder Stenosen gekennzeichnet ist.

Symptomatische, peri-anale Fisteln und Abszesse sollten zunächst drainiert werden, bevor eine anti-entzündliche Behandlung eingeleitet wird. Antibiotika wirken zunächst symptomatisch, ein dauerhafter Fistelverschluss wird hiermit jedoch nicht erreicht. Hierfür stehen Azathioprin/6Mercaptopurin sowie insbesondere anti-TNF Antikörper zur Verfügung. Gegebenenfalls ist eine zusätzliche chirurgische Intervention notwendig.

Nach dem Fistelverschluss wird eine Erhaltungstherapie über mindestens ein Jahr empfohlen. Hierzu eignen sich entweder Azathioprin/6-Mercaptopurin, Infliximab, Adalimumab, eine SetonDrainage oder eine Kombination aus medikamentöser Behandlung und Drainage. Bei nicht-perianaleperi-analen Fisteln ist eine Resektion des betroffenen Darmsegmentes oftmals unerlässlich.

Zur Behandlung von Stenosen stehen anti-entzündliche Medikamente (jedoch nur bei entzündlichen Stenosen), die endoskopische Ballondilatation sowie die Chirurgie zur Verfügung. Bei Verdacht auf entzündlich-bedingte Stenosen sollte zunächst ein kurzzeitiger Therapieversuch mit intravenösen Steroiden oder Infliximab durchgeführt werden. Ein Therapieversagen spricht für das Vorliegen einer fibrotischen Striktur und es muss entweder eine endoskopische Ballondilatation oder eine chirurgische Behandlung mittels Strikturoplastik oder Resektion des betroffenen Darmabschnittes durchgeführt werden. Bei fibrotischen Stenosen ist eine medikamentöse Behandlung nicht sinnvoll. 


\section{Lernfragen}

1. Ein dauerhafter Fistelverschluss wird häufig erreicht durch (mehrere Antworten richtig):
A: Infliximab
B: Aminosalicylate
C: Adalimumab
D: Prednison
E: Azathioprin

Antworten A, C und E sind richtig

2. Beim akuten Auftreten intestinaler Passagestörungen bei Morbus Crohn Patienten können folgende Massnahmen sinnvoll sein (mehrere Antworten richtig):

A: Intravenöse Steroide

B: Azathioprin

C: Endoskopische Ballondilatation

D: Infliximab

E: Strikturoplastik/Resektion des betroffenens Abschnittes

Antworten A, C, D und E sind richtig. 


\section{Korrespondenzadresse:}

PD Dr. med. Michael Scharl, Klinik für Gastroenterologie und Hepatologie, Department für Innere Medizin, Universitätsspital Zürich, Rämistrasse 100, 8091 Zürich, Schweiz. Telefon: +4144-255-9519, Fax: +41-44-255-9497, E-mail: michael.scharl@usz.ch 


\section{Bibliographie}

1. Cosnes J, Cattan S, Blain A, et al. Long-term evolution of disease behavior of Crohn's disease. Inflamm Bowel Dis. 2002;8:244-250

2. Schwartz DA, Loftus EV, Jr., Tremaine WJ, et al. The natural history of fistulizing Crohn's disease in Olmsted County, Minnesota. Gastroenterology. 2002;122:875-880

3. Sandborn WJ, Fazio VW, Feagan BG, et al. AGA technical review on perianal Crohn's disease. Gastroenterology. 2003;125:1508-1530

4. Bataille F, Klebl F, Rummele P, et al. Morphological characterisation of Crohn's disease fistulae. Gut. 2004;53:1314-1321

5. Nielsen $\mathrm{OH}$, Rogler G, Hahnloser D, et al. Diagnosis and management of fistulizing Crohn's disease. Nat Clin Pract Gastroenterol Hepatol. 2009;6:92-106

6. Van Assche G, Dignass A, Reinisch W, et al. The second European evidence-based Consensus on the diagnosis and management of Crohn's disease: Special situations. J Crohns Colitis. 2010;4:63-101

7. Pascua M, Su C, Lewis JD, et al. Meta-analysis: factors predicting post-operative recurrence with placebo therapy in patients with Crohn's disease. Aliment Pharmacol Ther. 2008;28:545-556

8. Vavricka SR, Rogler G. Fistula treatment: The unresolved challenge. Dig Dis. 2010;28:556-564

9. Bernstein LH, Frank MS, Brandt LJ, et al. Healing of perineal Crohn's disease with metronidazole. Gastroenterology. 1980;79:599 
10. West RL, van der Woude CJ, Hansen BE, et al. Clinical and endosonographic effect of ciprofloxacin on the treatment of perianal fistulae in Crohn's disease with infliximab: a doubleblind placebo-controlled study. Aliment Pharmacol Ther. 2004;20:1329-1336

11. Pearson DC, May GR, Fick GH, et al. Azathioprine and 6-mercaptopurine in Crohn disease. A meta-analysis. Ann Intern Med. 1995;123:132-142

12. Present DH, Rutgeerts $\mathrm{P}$, Targan S, et al. Infliximab for the treatment of fistulas in patients with Crohn's disease. N Engl J Med. 1999;340:1398-1405

13. Sands $\mathrm{BE}$, Anderson $\mathrm{FH}$, Bernstein $\mathrm{CN}$, et al. Infliximab maintenance therapy for fistulizing Crohn's disease. N Engl J Med. 2004;350:876-885

14. Colombel JF, Sandborn WJ, Rutgeerts P, et al. Adalimumab for maintenance of clinical response and remission in patients with Crohn's disease: the CHARM trial. Gastroenterology. $2007 ; 132: 52-65$

15. Colombel JF, Schwartz DA, Sandborn WJ, et al. Adalimumab for the treatment of fistulas in patients with Crohn's disease. Gut. 2009;58:940-948

16. Vavricka SR, Schoepfer AM, Bansky G, et al. Efficacy and safety of certolizumab pegol in an unselected crohn's disease population: 26-week data of the FACTS II survey. Inflamm Bowel Dis. 2011;17:1530-1539

17. Mahadevan U, Marion JF, Present DH. Fistula response to methotrexate in Crohn's disease: a case series. Aliment Pharmacol Ther. 2003;18:1003-1008

18. Sandborn WJ. Cyclosporine therapy for inflammatory bowel disease: definitive answers and remaining questions. Gastroenterology. 1995;109:1001-1003

19. Ng SC, Arebi N, Kamm MA. Medium-term results of oral tacrolimus treatment in refractory inflammatory bowel disease. Inflamm Bowel Dis. 2007;13:129-134 
20. Schoepfer AM, Safroneeva E, Vavricka SR, et al. Treatment of fibrostenotic and fistulizing Crohn's disease. Digestion. 2012;86 Suppl 1:23-27 


\section{Abbildungslegenden:}

Abbildung 1: Der Therapiealgorithmus bei Patienten mit kompliziertem peri-analem Morbus Crohn.

Abbildung 2: Der Therapiealgorithmus bei Patienten mit fibro-stenotischem Morbus Crohn. 